\title{
Hemisection: A Savior for Hopeless Teeth
}

\author{
${ }^{1}$ Prachi Mittal, ${ }^{2}$ Ashwini B Prasad, ${ }^{3}$ Deepak Raisingani, ${ }^{4}$ Amit, ${ }^{5}$ Purav Mehta, ${ }^{6}$ Dileep Soni, ${ }^{7}$ Deeksha Khurana
}

\begin{abstract}
Hemisection refers to the sectioning of a molar tooth with the removal of unrestorable root (along with the crown part) which maybe affected by periodontal, endodontic, structural (cracked roots), or caries. The treatment strategy involves a combined periodontal, endodontic and prosthodontic assessment and evaluation because in hemisection, like any other treatment, appropriate case selection is the most important factor determining the long-term success.
\end{abstract}

Keywords: Endodontic, Hemisection, Mandibular molar, Periodontal.

How to cite this article: Mittal $P$, Prasad $A B$, Raisingani $D$, Amit, Mehta P, Soni D, Khurana D. Hemisection: A Savior for Hopeless Teeth. J Mahatma Gandhi Univ Med Sci Tech 2016;1(1):30-34.

Source of support: Nil

Conflict of interest: None

\section{INTRODUCTION}

Advances in all phases of dentistry have come up with treatment modalities which is providing opportunity for the patients to maintain a functional dentition rather than compromising it. ${ }^{1}$ Retaining molars with extensive decay and compromised periodontal status has always been challenging and the treatment options, in the past, were limited to dental extraction and replacement. With the advancements, it has become possible to retain such teeth in whole or in part. Nevertheless, treatment strategy involves a combined periodontal, prosthodontic and endodontic assessment for proper case selection so that it ensures a stronger survival. ${ }^{2}$ Therapeutic measures performed to ensure retention of teeth vary in complexity.

Hemisection (removal of one root) is a procedure which involves removing significantly compromised root

\footnotetext{
${ }^{1,4-6}$ Senior Lecturer, ${ }^{2}$ Reader, ${ }^{3}$ Professor and Head, ${ }^{7}$ Resident

1-3,6,7 Department of Conservative Dentistry and Endodontics Mahatma Gandhi Dental College and Hospital, Jaipur Rajasthan, India

${ }^{4}$ Department of Oral and Maxillofacial Surgery, Mahatma Gandhi Dental College and Hospital, Jaipur, Rajasthan, India

${ }^{5}$ Department of Conservative Dentistry and Endodontics Ahmedabad Dental College, Ahmedabad, Gujarat, India

Corresponding Author: Prachi Mittal, Senior Lecturer Department of Conservative Dentistry and Endodontics Mahatma Gandhi Dental College and Hospital, Jaipur, Rajasthan India, Phone: +91 9829084781, e-mail: drprachi87@yahoo.com
}

structure and the associated coronal structure through deliberate excision. ${ }^{3}$ It is possible to utilize a segment of multirooted tooth as a healthy abutment even though retention of the entire tooth is impossible due to advanced periodontal disease, carious lesion or endodontic failure. The treatment goal is preservation of remaining tooth structure and restoration of the function.

\section{CASE REPORTS}

\section{Case 1}

A 25 years old man reported with the complaint of pain and mobility of right mandibular first molar. His medical history was noncontributory. On examination, the tooth was sensitive to percussion and revealed grade 1+ mobility. On probing the area, there was a $10 \mathrm{~mm}$ deep periodontal pocket around the distal root of the tooth. On radiographic examination, vertical bone loss was evident surrounding the distal root along with horizontal bone loss in the middle and coronal third along with root caries. The bony support of mesial root was completely intact. It was decided that the distal root should be hemisected after completion of endodontic therapy of the tooth. The working length was determined and both the mesial canals were prepared using crowndown technique (Rotary Protaper, Dentsply Maillefer). The canals were filled with Protaper F1 gutta-percha and the chamber was filled with composite (Fig. 1) to maintain a good seal and allow interproximal area to be properly contoured during surgical separation. Under local anesthesia, full thickness flap was reflected after giving a crevicular incision from first premolar to second molar. Upon reflection of the flap, the bony defect along the distal root became quite evident. All chronic inflammatory tissue was removed with curettes to expose the bone. The vertical cut method was used to resect the crown (Figs 2 and 3). A long shank tapered fissure carbide bur was used to make vertical cut toward the bifurcation area. A fine probe was passed through the cut to ensure separation. The distal root was extracted (Figs 4 to 6 ) and the socket was irrigated adequately with sterile saline to remove bony chips. The furcation area was trimmed to ensure that no spicules were present to cause further periodontal irritation. Scaling and root planning of the root surfaces, which became accessible on removal of distal root was done. The extraction site was irrigated and 


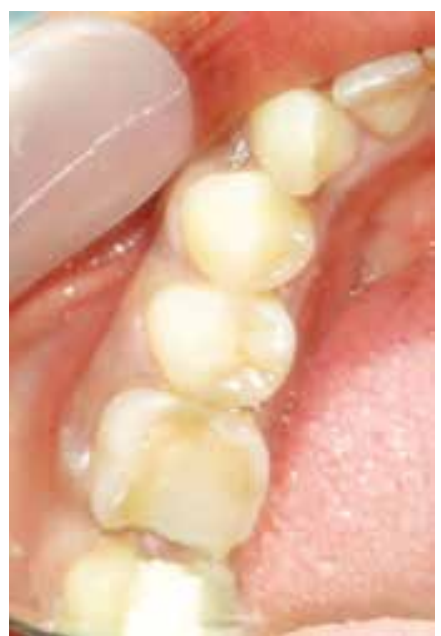

Fig. 1: Preoperative endodontically treated teeth and composite filled mandibular right 1st molar

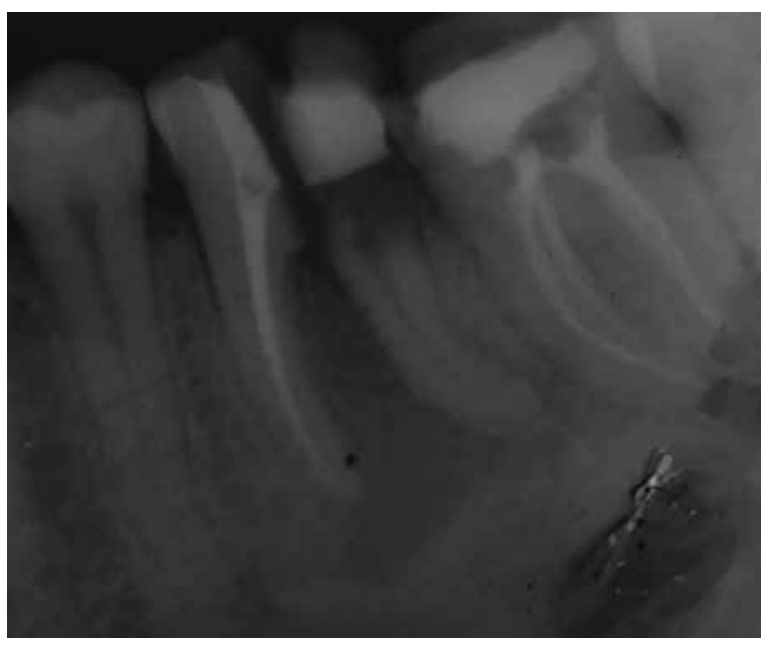

Fig. 3: Preoperative radiographic view

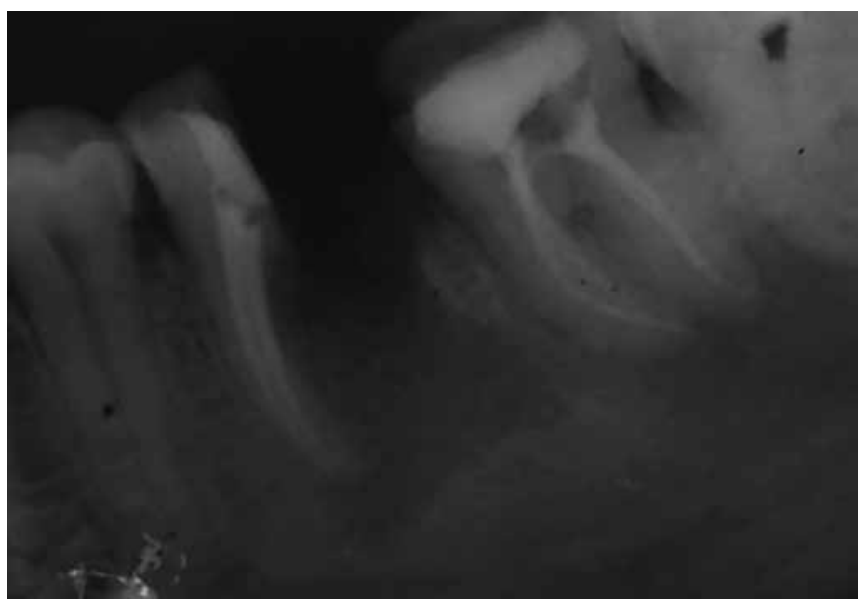

Fig. 5: Postoperative radiographic view after removal of distal fragment

debrided. Bioactive glass was placed as graft material for ridge preservation. The flap was then repositioned and sutured with 3/0 black silk sutures. The occlusal table

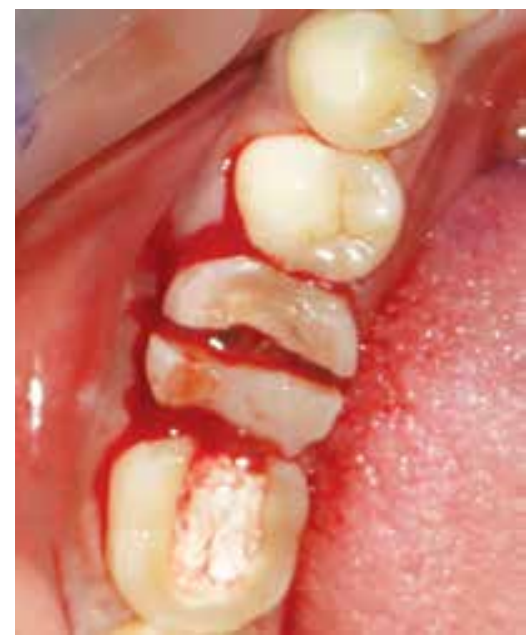

Fig. 2: Hemisectioning of tooth in mesial and distal halves

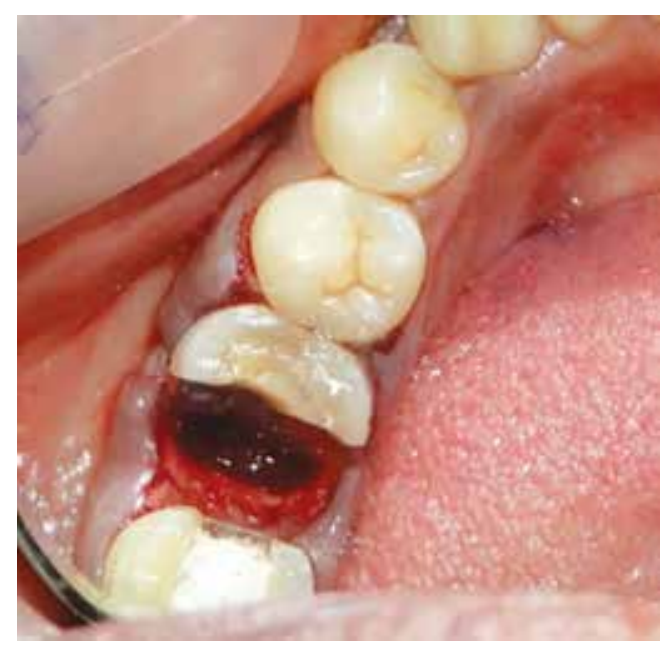

Fig. 4: Sectioned distal half of the removed tooth

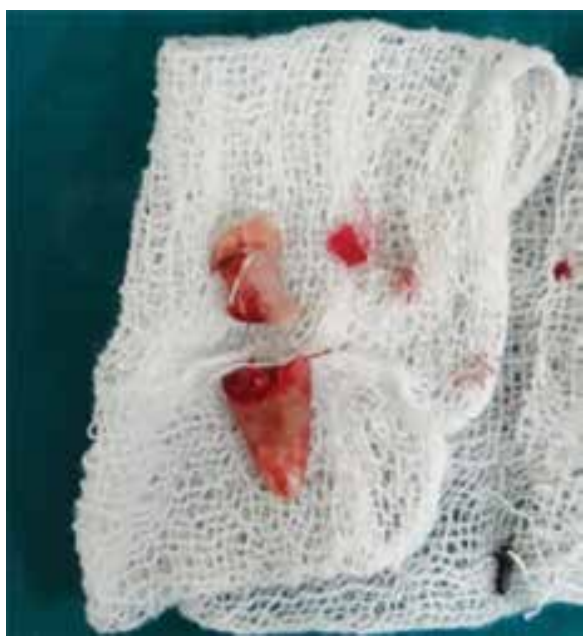

Fig. 6: Extracted distal half of tooth

was minimized to redirect the forces along the long axis of the mesial root (Fig. 7). After healing of the tissues, impression was made, wax pattern was made in die was 


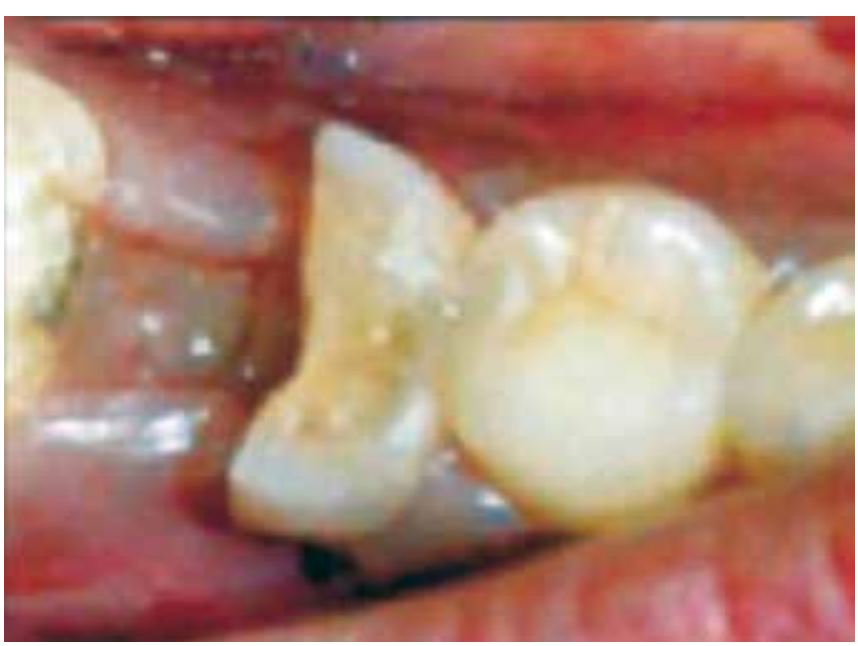

Fig. 7: Postoperative view

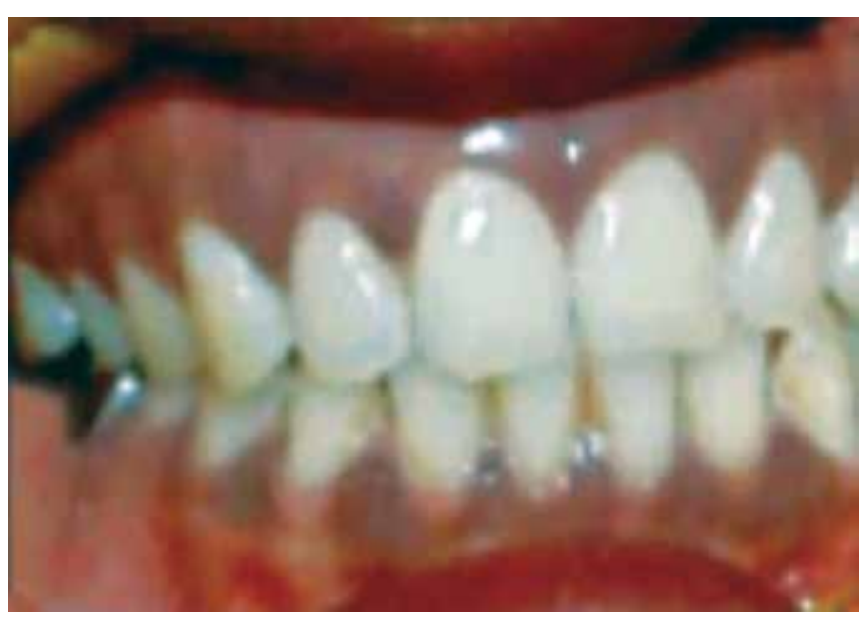

Fig. 9: Final prosthesis cemented

fabricated (Fig. 8) and after that final prosthesis, i.e, fixed bridge involving retained mesial half and mandibular second molar with sanitary pontic was given (Fig. 9).

\section{Case 2}

A 45 years old man in good health reported with the complaint of decayed tooth with intermittent pain in lower right teeth region. On clinical examination, a large caries lesion was found in the right 1st mandibular molar (Fig. 10), the tooth was sensitive to percussion and revealed grade 1 mobility. On probing the area, there was a $8 \mathrm{~mm}$ deep periodontal pocket around the mesial root of the tooth. On radiographic examination, vertical bone loss was evident surrounding the mesial root and involving the furcation area (Fig. 11). The distal root was in good condition with sound periodontal support. The diagnosis of pulp necrosis with periodontal pocket was given in the involved tooth, i.e, 46 . The patient did not wish to have the tooth removed. Thus, it was decided that the mesial root should be hemisected after completion of endodontic therapy of the tooth followed by placement of final prosthesis. The option of hemisection was

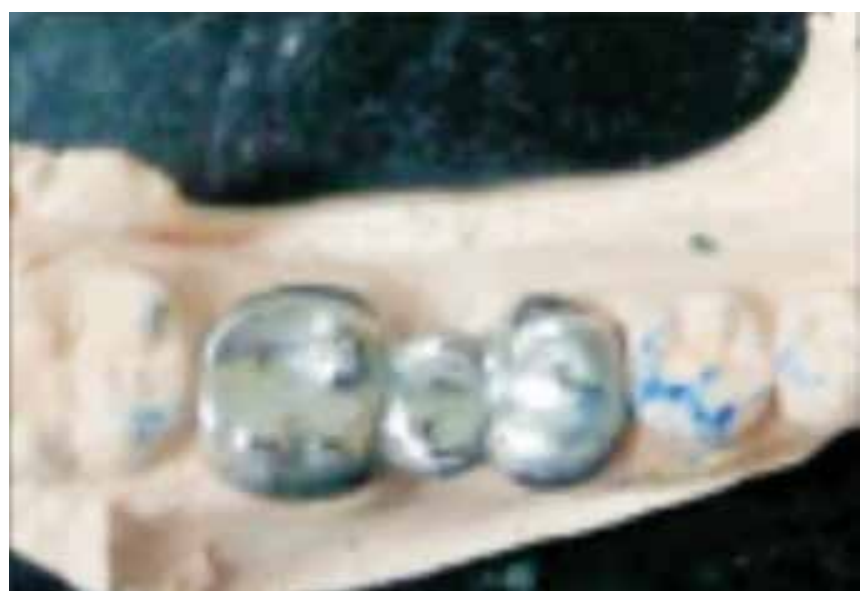

Fig. 8: Die pattern of final prosthesis

discussed with all the risks explained to the patient. The patient agreed to this treatment option. In the following appointment, the endodontic access was made, the working length was determined and the canals were biomechanically prepared using crowndown technique (Rotary Protaper, Dentsply Maillefer). The canals were filled with Protaper F1 Gutta-percha, and the chamber was filled with composite to maintain a good seal and allow interproximal area to be properly contoured during surgical separation (Fig. 12). Under local anesthesia, full thickness flap was reflected after giving a crevicular incision from first premolar to second molar. Upon reflection of the flap, the bony defect along the mesial root became quite evident. All chronic inflammatory tissue was removed with curettes to expose the bone. The vertical cut method was used to resect the crown (Fig. 13). A long shank tapered fissure carbide bur was used to make vertical cut toward the bifurcation area. A fine probe was passed through the cut to ensure separation. The mesial root was extracted and the socket was irrigated adequately with sterile saline to remove bony chips and amalgam debris (Figs 14 and 15). The furcation area was trimmed to ensure that no spicules were present and any defects on the sound distal root were smoothened. Scaling and root planning of the root surfaces, which became accessible on removal of mesial root was done. The extraction site was irrigated and debrided and the flap was then repositioned and sutured with $3 / 0$ black silk sutures (Fig. 16). The surgical site was then allowed to heal with no occlusal stress placed on the root for 4 weeks. After healing of the tissues, fixed bridge involving retained distal half and mandibular second molar with sanitary pontic was given (Fig. 17).

\section{DISCUSSION}

Success of root resection procedures depend, to a large extent, on proper case selection. It is important to consider 


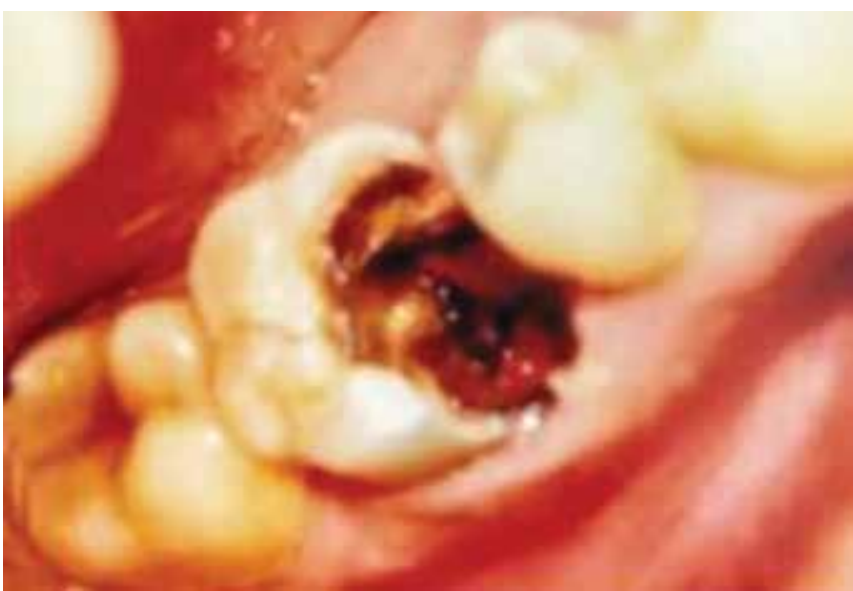

Fig. 10: Preoperative view

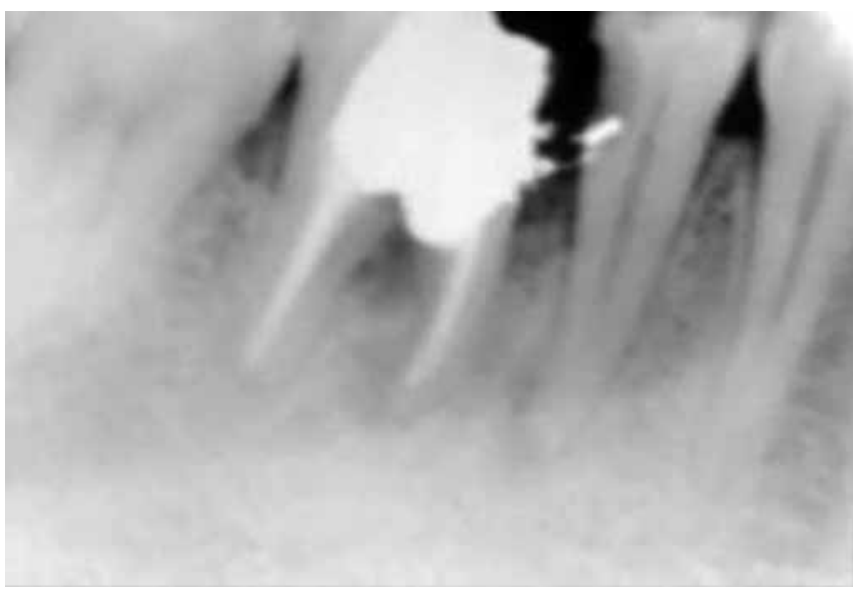

Fig. 12: Obturation

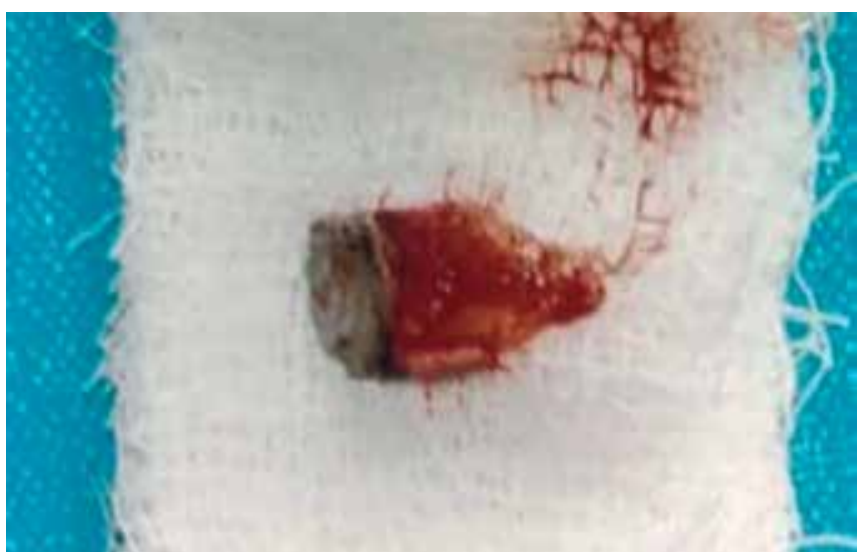

Fig. 14: Tooth fragment

the following factors before deciding to undertake any of the resection procedures. ${ }^{4}$

- Advanced bone loss around one root with acceptable level of bone around the remaining roots.

- Angulation and position of the tooth in the arch. A molar, i.e., buccally, lingually, mesially or distally titled, cannot be resected.

- Divergence of the roots - teeth with divergent roots are easier to resect. Closely approximated or fused roots are poor candidates.

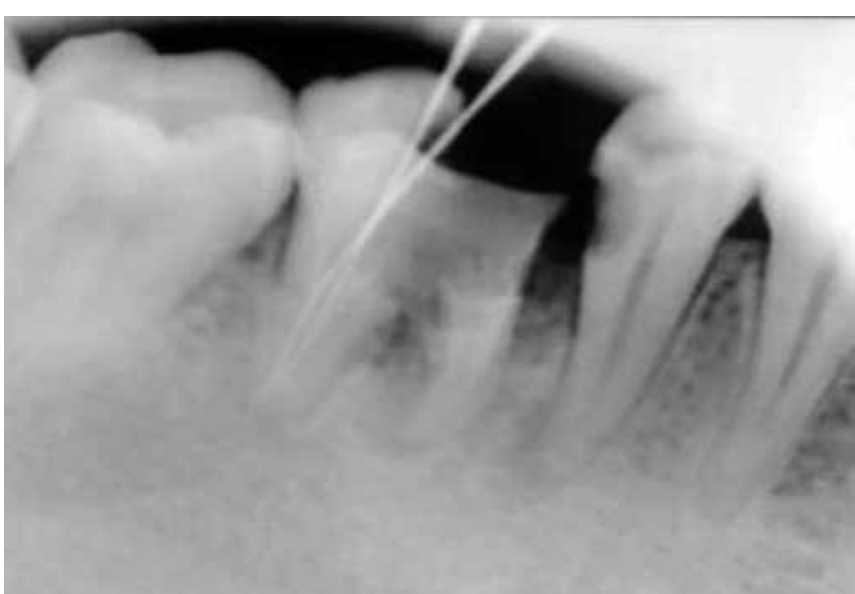

Fig. 11: Diagnostic IOPA

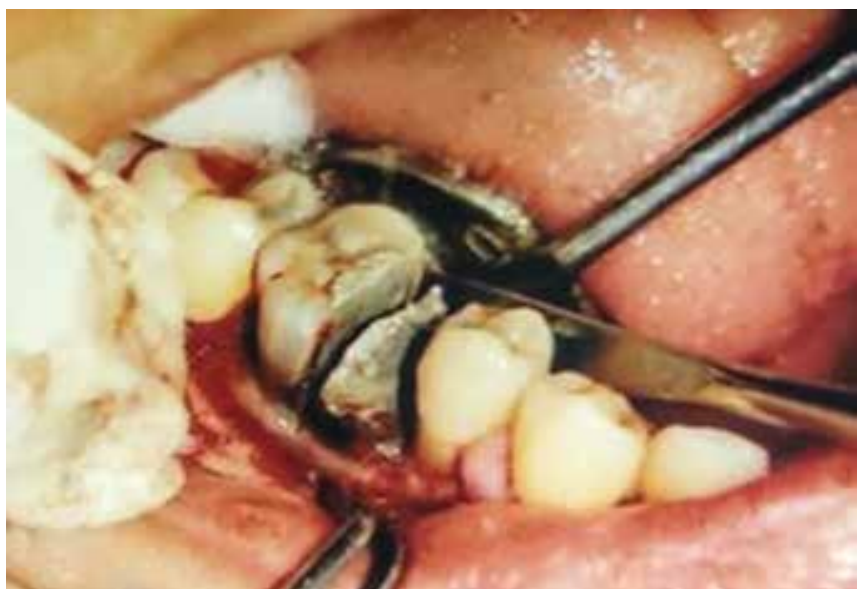

Fig. 13: Hemisection

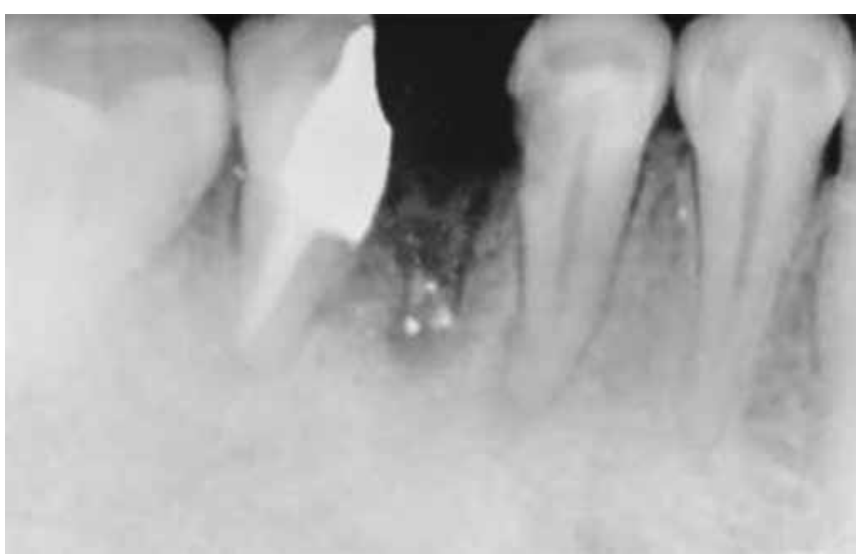

Fig. 15: Postoperative IOPA view

- Length and curvature of roots - long and straight roots are more favorable for resection than short, conical roots.

- Feasibility of endodontics and restorative dentistry in the root/roots to be retained.

Some investigators reported that root-resected molars had $>90 \%$ survival rate Whereas other investigators reported that $30 \%$ of resected molars failed over a $10-$ year period. ${ }^{5}$ 


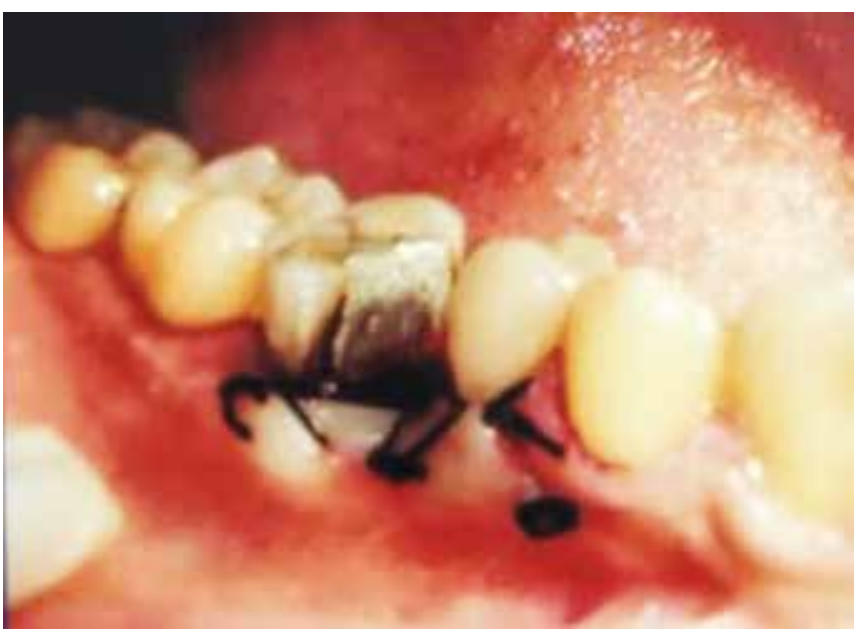

Fig. 16: Suture placed

In this case, bioactive glass was placed as the graft material which consists of sodium and calcium salts, phosphate and silicon dioxide. The particles measure from 90 to 170 microns. When this material comes in contact with tissue fluids, the surface of the particle becomes coated with hydroxycarbonate apatite, incorporates organic ground proteins, such as chondroitin sulfate and glycosaminoglycans and attracts osteoblasts that rapidly form bone.

Hemisection has been used successfully to retain teeth with furcation involvement. However, there are few disadvantages associated with it. As with any surgical procedure, it can cause pain and anxiety. Root surfaces that are reshaped by grinding in the furcation or at the site of hemisection are more susceptible to caries. Often a favorable result may be negated by decay after treatment. ${ }^{6}$ Failure of endodontic therapy due to any reason will cause failure of the procedure. In addition, when the tooth has lost part of its root support, it will require a restoration to permit it to function independently or to serve as an abutment for a splint or bridge. Unfortunately, a restoration can contribute to periodontal destruction, if the margins are defective or if non-occlusal surfaces do not have physiologic form. Also, an improperly shaped occlusal contact area may convert acceptable forces into destructive forces and predispose the tooth to trauma from occlusion and ultimate failure of hemisection. Contraindications include the presence of a strong abutment tooth adjacent to the proposed hemisection, which could act as an abutment to prosthesis. ${ }^{7}$ The remaining root may be inoperable for the necessary root canal treatment. Also, fusion or proximity of the roots may prevent their separation. Hemisection allows for physiologic tooth mobility of the remaining root, which is thus a more suitable abutment for fixed partial dentures than an osseointegrated counterpart. ${ }^{8}$

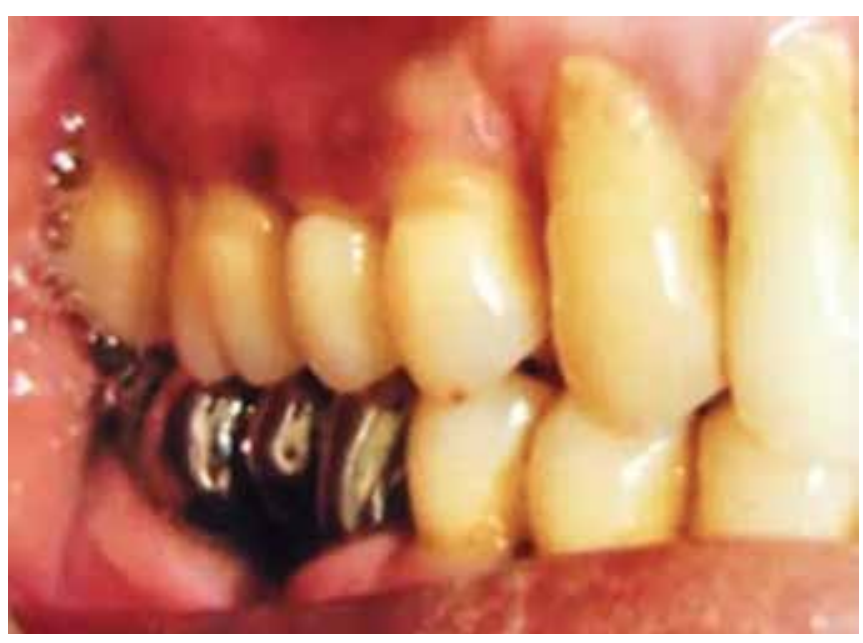

Fig. 17: Placement of fixed restoration

\section{CONCLUSION}

The prognosis for hemisection is the same as for routine endodontic procedures provided. ${ }^{9}$

That case selection has been correct.

The endodontics has been performed adequately, and

The restoration is of an acceptable design relative to the occlusal and periodontal needs of the patient.

Root amputation and hemisection should be considered as another weapon in the arsenal of the dental surgeon, determined to retain and not remove the natural teeth. ${ }^{10}$ With recent refinements in endodontics, periodontics, and restorative dentistry. Hemisection has received acceptance as a conservative and dependable dental treatment and teeth so treated have endured the demands of function.

\section{REFERENCES}

1. Parmer G, Vashi P. Hemisection: a case report and review Endodontology 2003:15.

2. Babaji $P$, Sihag $T$, Chaurasia VR, Senthilnathan S. Hemisection: a conservative management of a periodontally involved molar tooth in a young patient. J Nat Sci Biol Med 2015 JanJun;6(1):253-255.

3. Saad MN, Moreno J, Crawford C. Hemisection as an alternative treatment for decayed multirooted terminal abutments: a case report. JCDA.www.cda-adc.ca/jcda. 2009 June;75(5).

4. Weine FS: Endodontic therapy. Mosby Co. St. Louis,5th ed 2006.

5. Endodontics Ingle, Elsevier BC. 5th 2002 edition

6. Carranza and Newman. Clinical Periodontology, 8th edi WB Saunders Co. Philadelphia.

7. Saraf AA, Patil AC. Hemisection. World J Dent 2013;4(3): 180-187.

8. Shah JJ, Baburaj MD, Pimpale S. Hemisection: split to benefit- a case report. IJSS Case Reports \& Review. 2015 Nov;2(6).

9. Chopra P, Gupta A, Tiwari R. Salvaging of hopeless teeth by an interdisciplinary approach. J Orofac Res 2015;5(4):155-159.

10. Verma PK, Srivastava R, Baranwal HC, Gautam A. A ray of hope for the hopeless; hemisection of mandibular molar with socket preservation. Dent Hypotheses 2012;3:159-163. 